\title{
Diseño de una máquina prototipo para la separación y remoción de partículas ferromagnéticas de neumáticos triturados
}

\author{
(Design of a prototype machine for separation and removal of \\ ferromagnetic particles from shredded tires)
}

\author{
Joao Bárzaga ${ }^{1}$, Yoandrys Morales ${ }^{1}$, Marioxy Morales ${ }^{1}$, Jhon Martínez ${ }^{2}$, Klever Paccha ${ }^{2}$ \\ ${ }^{1}$ Universidad Técnica de Cotopaxi Extensión La Maná, La Maná, Ecuador \\ ${ }^{2}$ Agroaereo, Valencia, Ecuador \\ joao.barzaga5406@utc.edu.ec,yoandrys.morales@utc.edu.ec,marioxy.morales@utc.edu.ec, \\ jhon99martinez@hotmail.com,pacchakleverv@gmail.com
}

\begin{abstract}
Resumen: La presente investigación tiene como objetivo el diseño de una máquina prototipo para separación y remoción de partículas ferromagnéticas de neumáticos triturados. Para la selección del prototipo a diseñar se analizaron los modelos existentes. El diseño se realizó mediante el software Autodesk Inventor que permite además realizar el análisis de elementos finitos. Por otra parte, se realizaron los cálculos para la selección del motor, potencia y momento torsor. Los resultados del trabajo presentan que el modelo diseñado tiene al acero AISI 1020 para la estructura inferior y superior, el acero AISI 304 para el tambor magnético y se utilizaron imanes cerámicos $\mathrm{C} 8$ debido al bajo costo respecto a otros. El moto-reductor calculado posee una velocidad de $1426 \mathrm{~min}^{-1}$ y el reductor es 40:1. La potencia requerida para el proceso de remoción fue de 140,65 W, además, el torque obtenido fue de 38,43 N.m. En el cálculo de las tensiones de Von Mises se obtuvo un máximo de 49,1 N, inferior al límite elástico del material. Asimismo, el máximo valor en el desplazamiento resultante se encuentra en el soporte de las chumaceras con $7,7 \cdot 10^{-5}$ $\mathrm{m}$, lo que demuestra que no existen deformaciones. Por último, los resultados del coeficiente de seguridad muestran valores entre 5,6 y 15 , esto demuestra que la relación entre tensión última y tensión admisible están distantes.
\end{abstract}

Palabras clave: diseño, máquina prototipo, separación, remoción, elementos finitos.

\begin{abstract}
The present research aims to design a prototype machine for separation and removal of ferromagnetic particles from shredded tires. For the selection of the prototype to be designed, the existing models were analyzed. The design was made using Autodesk Inventor software that also allows for finite element analysis. On the other hand, the calculations for the selection of the motor, power and torque were carried out. The results of the work show that the designed model has AISI 1020 steel for the lower and upper structure, AISI 304 steel for the magnetic drum and C8 ceramic magnets were used due to the low cost compared to others. The motor-reducer calculated has a speed of $1426 \mathrm{~min}^{-1}$ and the reducer is 40: 1 . The power required for the removal process was $140.65 \mathrm{~W}$, in addition, the torque obtained was $38.43 \mathrm{~N} \cdot \mathrm{m}$. In the calculation of the Von Mises stresses, a maximum of $49.1 \mathrm{~N}$ was obtained, lower than the elastic limit of the material. Likewise, the maximum value in the resulting displacement is found in the bearing support with $7.7 \cdot 10^{-5}$ $\mathrm{m}$, which shows that there are no deformations. Finally, the results of the safety factor show values between 5.6 and 15, this shows that the relationship between ultimate stress and allowable stress are distant.
\end{abstract}

Keywords: design, prototype machine, separation, removal, finite elements. 


\section{INTRODUCCIÓN}

Actualmente el tema de la reutilización de los recursos cobra mayor relevancia, tomando en cuenta que es de vital importancia incorporar estrategias para establecer direccionamientos que permitan un sistema integral de actividades. Se ha encontrado una tendencia hacia los materiales reciclados en el país y de esta forma podemos utilizar el material de llantas usadas, para obtener polvo o arena plástica para la elaboración de productos innovadores.

Debido al gran aumento del número de automóviles en todo el mundo, la acumulación de enormes volúmenes de neumáticos de desecho se ha convertido en un importante problema de gestión de residuos. Una gran cantidad de neumáticos de caucho usados se acumulan en el mundo cada año [1, 2]. La gestión de residuos, como todo esfuerzo medioambiental, involucra factores estresantes y receptores. Los factores estresantes son los agentes que deben eliminarse, disminuirse o gestionarse de otro modo, como los desechos en sí o ciertos componentes de los desechos, por ejemplo, metales pesados, compuestos orgánicos, microbios o energía. Los receptores, por definición, reciben el daño causado por los factores estresantes [3].

El mercado de materiales de neumáticos reciclados ha crecido exponencialmente a lo largo de los años. Han aumentado el mérito de los productos principalmente porque han demostrado que son los más apropiados y efectivos para una aplicación particular. El hecho de que el material sea de fácil acceso y, a menudo, el más rentable en comparación con los materiales tradicionales también ha contribuido a la expansión. Los mismos pueden ser convertidos en alfombras, bloques de caucho para parques infantiles, textiles y asfalto, césped sintético etc. [4].

No importa dónde se produzca el neumático, todos los neumáticos contienen cuatro grupos de materiales fundamentales: cauchos naturales o sintéticos, negros de humo / sílices, material de refuerzo (metales / textiles) y facilitadores. Un neumático puede llegar al final de su vida útil en carretera casi en cualquier punto después de la producción y la iniciación en carretera. Una vez que un neumático ha sido retirado de forma permanente de un vehículo sin posibilidad de ser devuelto a la carretera, se define como "residuo". A partir de ese momento entra en un sistema de gestión de residuos [4].

El sistema de gestión de residuo puede ser catalogado cómo el proceso de recuperación de los componentes del neumático para su reutilización. Para lograr este proceso se requiere de equipamiento que corte la banda lateral, que pulverice y separe las partículas metálicas presentes. A nivel industrial existen procedimientos que permiten la separación de las partículas de acero que contienen los granos de caucho triturado, esto permite dar utilidad a los neumáticos fuera de uso (NFU).

Los tratamientos de reciclaje de llantas van desde los más simples dispositivos mecánicos de corte o compresión hasta sofisticados y complejos procesos químicos, mecanoquímicos y /o térmicos multifásicos, que superan muchos de los principales obstáculos inherentes al reciclaje de cauchos termoestables. Existen cuatro niveles básicos de tratamiento. El material que se obtiene luego de los cuatro niveles de tratamientos se clasifica en seis categorías: cortes, triturados, astillas, granulados, polvos y polvos finos. La mayoría de los polvos finos resultan del tercer y cuarto nivel [4].

Los separadores magnéticos que eliminan fragmentos metálicos se utilizan generalmente para proteger equipos, tales como trituradoras, pulverizadores, etc. Son normalmente aplicados sobre materiales secos o sobre materiales que contengan solamente humedad superficial. Por ello, se requiere de un diseño que cumpla con los parámetros deseados.

El proceso de diseño de elementos de máquinas requiere de la necesidad del equipo, además de sus componentes para luego realizar los cálculos pertinentes. Por tales razones que usualmente requieren de largos tiempos, han surgido programas para el diseño asistido por computadora (CAD). 
El CAD y la ingeniería asistida por computadora (CAE) se han aplicado ampliamente en las prácticas del diseño de ingeniería. Los diseños de ingeniería se clasifican en términos del nivel de creatividad, se comparan los roles de los diseñadores y se discute el impacto de las técnicas asistidas por computadora en la creatividad y efectividad del diseño [5].

La ingeniería asistida por computadora se convierte en una herramienta ideal cuando se evalúa un gran número de construcciones para determinar la mejor solución sujeta a determinadas restricciones. El análisis de elementos finitos (FEA) es una de las técnicas más importante dentro de esta, el modelo de este análisis está compuesto por nodos y elementos en todo su dominio. Posee la capacidad superior de resolver problemas diversificados para geometrías complejas es uno de los atributos más importantes [5].

Los estudios realizados mediante FEA permiten la toma de decisiones respecto a los resultados y con ello, hacer cambios para lograr el modelo deseado. Ejemplo de lo anterior ha sido evidenciado en [6], donde se muestran soluciones con el uso de estas herramientas, lo que realza la importancia del modelado y el cálculo mediante elementos finitos. Por otra parte, en [7] plantean que la oportunidad de disponer de herramientas informáticas permite optimizar el diseño mecánico por minimización de las tensiones localizadas en piezas de máquinas con formas complejas combinadas.

Algunas investigaciones [8-11] muestran el uso de las herramientas CAD y FEA para el estudio de diferentes estructuras lo que permite tener respuestas acertadas de los problemas que se presentan.

La mayoría de los NFU del Cantón La Maná, una vez cumplida su vida útil en los vehículos pasan a formar parte de los vertederos controlados e incontrolados, pues los neumáticos tienen un impacto ambiental negativo. La manufacturación constante de neumáticos y la dificultad para reciclarlos después de ser utilizados se convierte en un problema ambiental que se debe controlar.

Teniendo en cuenta la inadecuada disposición de los neumáticos en basureros al aire libre, en los afluentes, rellenos sanitarios, la quema indiscriminada a cielo abierto, así como la falta de interés en la creación de políticas que fortalezcan la recaudación económicamente e implementación de manufacturas, con la tecnología adecuada para reconsiderar estos desechos y convertirlos nuevamente en materia prima útil. Por estas razones, la carrera Ingeniería Electromecánica perteneciente a la Universidad Técnica de Cotopaxi, extensión La Maná ha desarrollado un Proyecto Formativo dirigido a la creación de máquinas que permitan el reciclado de los neumáticos fuera de uso.

El proceso de la recuperación de la materia prima de los neumáticos tiene varios procesos que pasa desde el desprendimiento de las bandas laterales que poseen elementos metálicos, el corte en pequeños trozos y por último la separación del caucho y el metal. Por ende, cada proceso no puede obviarse, para así obtener un producto listo para su utilización en diferentes ámbitos. En base a lo expuesto anteriormente, la presente investigación tiene como objetivo, diseñar una máquina prototipo para la separación y remoción de partículas ferromagnéticas de neumáticos triturados.

\section{MATERIALES Y MÉTODOS}

En base a lo pretendido y conseguido de la investigación bibliográfica se determina que la velocidad de rotación del tambor debe estar dentro de los parámetros entre $30 \mathrm{~min}^{-1}$ a $50 \mathrm{~min}^{-1}$. Como se encuentra a disposición un motoreductor de $0,25 \mathrm{HP}$, que gira a una velocidad de 1426 $\min ^{-1}$, es necesario acoplar un sistema reductor con una relación de transmisión 40:1 el cual debe generar una velocidad en la salida de $35 \mathrm{~min}^{-1}$, siendo esta velocidad correcta por estar dentro de los parámetros investigados anteriormente. 


\subsection{Modelado del prototipo de máquina para la separación y remoción de partículas ferromagnéticas}

Para el modelado del prototipo, se utilizó el software de diseño asistido por computadora Autodesk Inventor que además permite realizar el análisis de elementos finitos. En la figura 1 se observa el modelo del tambor magnético desarrollado para el prototipo de máquina objeto de estudio. Este elemento permite recibir desde la tolva la mezcla de partículas metálicas y de caucho. En el interior de este se encuentra el eje fijo que sostiene el tambor interno con los elementos magnéticos.

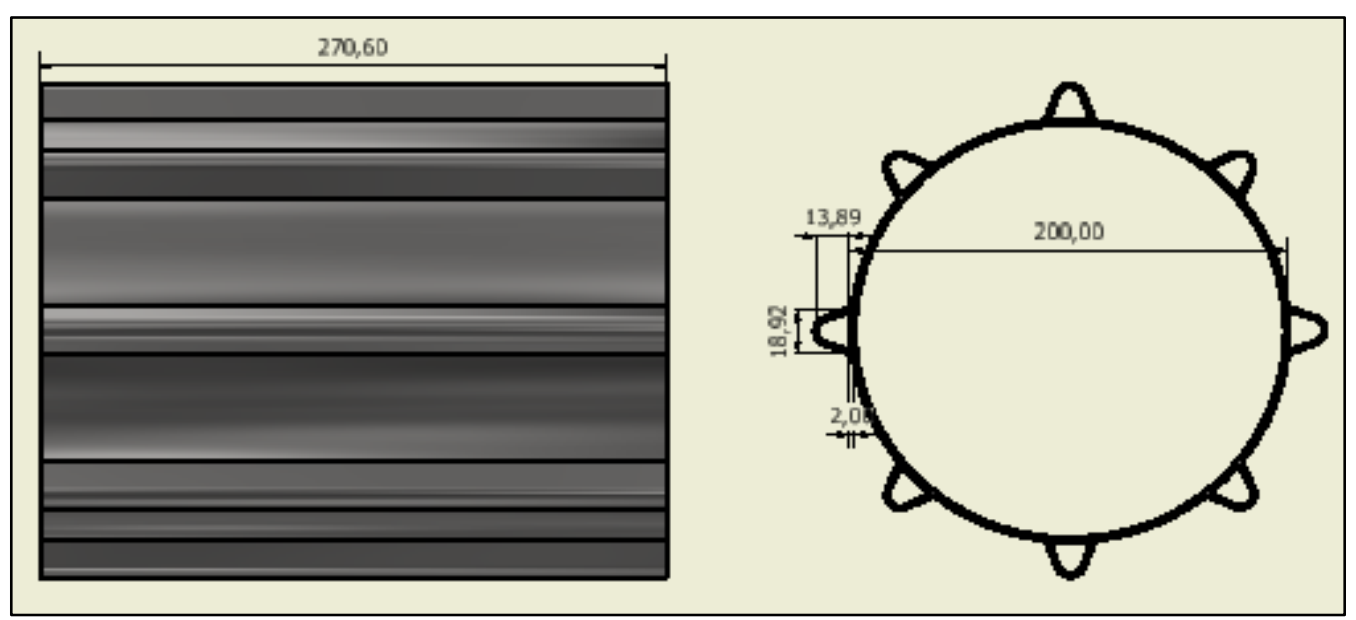

Figura 1. Plano de tambor magnético.

En la figura 2 se puede observar el modelo de tolva desarrollado. Algunos elementos que no se muestran y son de importancia en el prototipo de máquina son: el eje motriz, el eje que soporta los elementos magnéticos y las tapas correspondientes acopladas en los laterales de los tambores. Además, se encuentra la estructura soporte y la carcasa inferior donde las partículas de caucho van hacia un lado y el material metálico hacia el otro mediante una estructura acanalada.

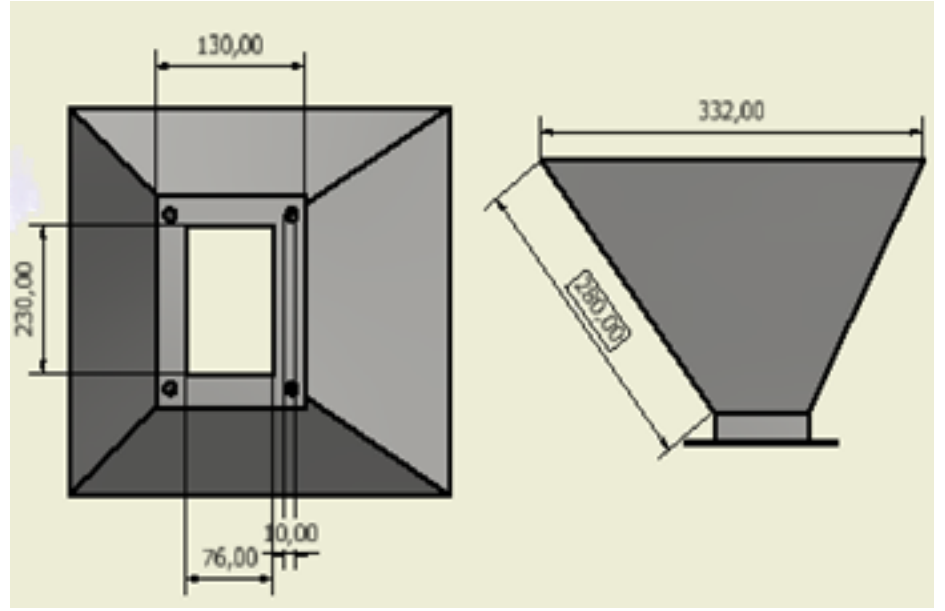

Figura 2. Plano de la tolva de alimentación.

En la figura 3 se muestra el modelo de ensamble de la máquina, en la misma se observa la posición de cada elemento. Se observa el moto-reductor, el sistema de encendido y apagado, el separador entre la tolva y la carcasa superior. 


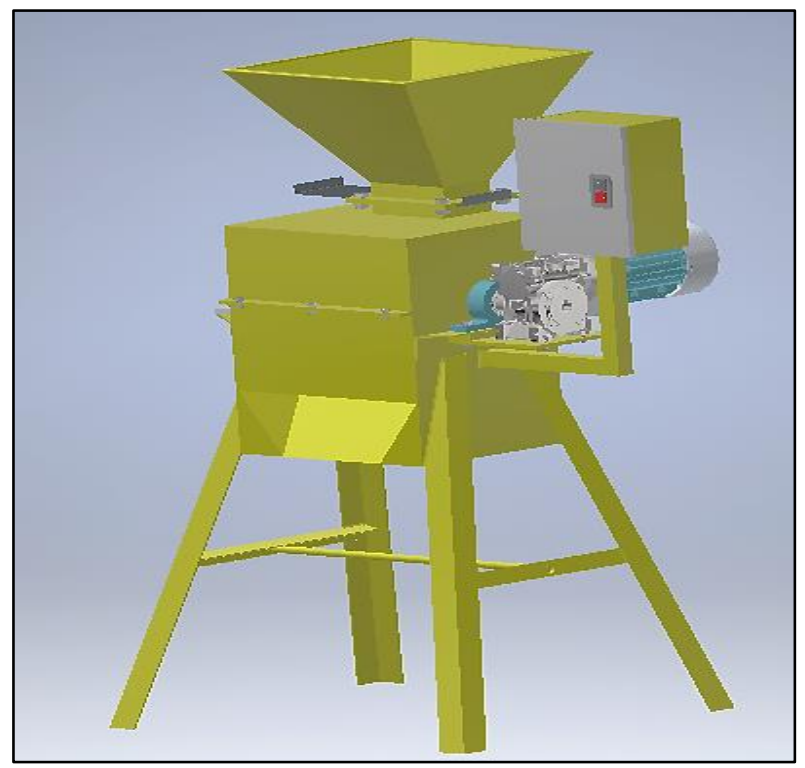

Figura 3. Modelo de ensamble de la máquina.

En la tabla 1 se pueden observar la masa de algunos componentes pertenecientes a la máquina antes mencionada.

Tabla 1. Valores en kilogramos de cada uno de los elementos.

\begin{tabular}{llll}
\hline Componente & Cantidad & Masa unitaria (kg) & Masa total (kg) \\
\hline Tambor rotacional & 1 & 1,63 & 1,63 \\
Tapa de cilindro giratorio & 2 & 4,32 & 4,32 \\
Eje porta masa magnética & 1 & 10,984 & 10,984 \\
Eje motriz & 1 & 0,43 & 0,43 \\
Pernos de sujeción total & 20 & 0,492 & 0,492 \\
Estructura soporte inferior & 1 & 15,08 & 15,08 \\
\hline
\end{tabular}

\subsection{Análisis de parámetros funcionales de la máquina}

En mecánica rotacional, la inercia rotacional desempeña un papel similar al de la masa en la mecánica lineal. De hecho, la inercia rotacional de un objeto depende de su masa. También depende de la distribución de esa masa respecto al eje de rotación.

Cálculo de la Inercia del tambor.

$$
\mathrm{I}_{\mathrm{x} 1}=\frac{1}{2} \mathrm{M}\left(\mathrm{r}_{\mathrm{e}}^{2}-\mathrm{r}_{\mathrm{i}}^{2}\right)=1,47 \cdot 10^{-2} \mathrm{~kg} \cdot \mathrm{m}^{2}
$$

Donde:

Masa del cilindro $(\mathrm{M})=1,63$ kilogramos $(\mathrm{kg})$

Radio exterior $\left(\mathrm{r}_{\mathrm{e}}\right)=0,1$ metros $(\mathrm{m})$

Radio menor $\left(\mathrm{r}_{\mathrm{i}}\right)=0,09$ metros $(\mathrm{m})$

Inercia $\left(I_{x 1}\right)=$ kilogramos sobre metros cuadrado $\left(\mathrm{kg} \cdot \mathrm{m}^{2}\right)$ 
Cálculo de la Inercia de la tapa del tambor.

$$
\mathrm{I}_{\mathrm{x} 2}=\frac{1}{2} \mathrm{Mr}^{2}=2,7 \cdot 10^{-2} \mathrm{~kg} \cdot \mathrm{m}^{2}
$$

Donde:

$$
\begin{aligned}
& \text { Masa de la tapa del tambor }(\mathrm{M})=2,16 \text { kilogramos }(\mathrm{kg}) \\
& \text { Radio exterior }(\mathrm{r})=0,112 \text { metros }(\mathrm{m}) \\
& \text { Inercia }\left(\mathrm{I}_{\mathrm{x} 2}\right)=\mathrm{kilogramos} \text { sobre metros cuadrado }\left(\mathrm{kg} \cdot \mathrm{m}^{2}\right)
\end{aligned}
$$

Cálculo de la Inercia del eje motriz.

El momento de inercia refleja la distribución de masa de un cuerpo o de un sistema de partículas en rotación, respecto a un eje de giro. El momento de inercia desempeña un papel análogo al de la masa inercial en el caso del movimiento rectilíneo y uniforme.

$$
\mathrm{I}_{\mathrm{x} 3}=\frac{1}{2} \mathrm{Mr}^{2}=2,77 \cdot 10^{-4} \mathrm{~kg} \cdot \mathrm{m}^{2}
$$

Donde:

$$
\begin{aligned}
& \text { Masa del eje motriz }(M)=0,43 \text { kilogramos }(\mathrm{kg}) \\
& \text { Radio exterior }(\mathrm{r})=0,0254 \text { metros }(\mathrm{m}) \\
& \text { Inercia }\left(\mathrm{I}_{\mathrm{x} 3}\right)=\text { kilogramos sobre metros cuadrado }\left(\mathrm{kg} \cdot \mathrm{m}^{2}\right)
\end{aligned}
$$

Cálculo de la Inercia total del sistema.

La inercia es la propiedad que tienen los cuerpos de permanecer en su estado de reposo relativo o movimiento relativo. Dicho de forma general, es la resistencia que opone la materia al modificar su estado de movimiento, incluyendo cambios en la velocidad o en la dirección del movimiento.

$$
\mathrm{I}_{\mathrm{T}}=\mathrm{I}_{\mathrm{x} 1}+\mathrm{I}_{\mathrm{x} 2}+\mathrm{I}_{\mathrm{x} 3}=4,2 \cdot 10^{-2} \mathrm{~kg} \cdot \mathrm{m}^{2}
$$

Cálculo del Torque o Par motor necesario.

El torque o momento torsor necesario para realizar el movimiento del sistema es importante para la selección del motor eléctrico. Teniendo en cuenta los datos que se utilizan en este tipo de máquinas se procede a presentar la siguiente ecuación.

$$
\mathrm{M}=\mathrm{I}_{\mathrm{T}} \cdot \alpha=38,43 \mathrm{~N} \cdot \mathrm{m}
$$

Datos

Aceleración angular $(\alpha)=915\left(\mathrm{~s}^{-2}\right)$

Inercia total del sistema $\left(\mathrm{I}_{\mathrm{T}}\right)=\left(\mathrm{kg} \cdot \mathrm{m}^{2}\right)$

Cálculo de la potencia del motor requerida en el sistema.

Se procede a realizar el cálculo de la potencia del motor mediante el momento torsor que ejerce el eje sometido a cargas externas, para determinar que el equipo trabaje sin sobre esfuerzos producidos por las cargas que interactúan en el eje, los cálculos se detallan a continuación:

Datos:

$$
\mathrm{P}=\mathrm{M} \cdot \omega=140,65 \mathrm{~W}=0,19 \mathrm{HP}
$$

Torque $(\mathrm{M})=38,43(\mathrm{~N} \cdot \mathrm{m})$

Velocidad angular $(\omega)=3,66\left(\mathrm{~s}^{-1}\right)$ 


\subsection{Análisis por elementos finitos aplicado a la estructura de la máquina}

El estudio del modelo del prototipo de máquina para la separación y remoción de partículas ferromagnéticas es necesario para conocer el comportamiento estructural de la misma, debido a que la estructura inferior soporta la carga de todos los elementos restantes. El estudio que se realiza es un análisis de tensiones estático, aplicado a la estructura inferior de la máquina prototipo. Según las condiciones del modelo, la parte inferior soporta las cargas de los elementos que componen el sistema rotatorio para la separación de las partículas ferromagnéticas, así como la parte superior.

\subsection{Análisis de las condiciones de contorno}

El material utilizado para el modelo del prototipo de máquina fue el acero AISI 1020, el cual es un acero de bajo contenido de carbono que puede ser mecanizado y de gran soldabilidad, además puede ser utilizado en la fabricación de maquinaria y construcción de piezas estructurales. En la tabla 2 se pueden observar las características físico-mecánicas del mismo.

Tabla 2. Propiedades del acero 1020.

\begin{tabular}{lll}
\hline Nombre & Acero AISI 1020 107 LC & \\
\hline General & Densidad de masa & $7870 \mathrm{~kg} \cdot \mathrm{m}^{-3}$ \\
& Límite de elasticidad & $260 \mathrm{MPa}$ \\
& Resistencia máxima a tracción & $441 \mathrm{MPa}$ \\
\multirow{2}{*}{ Tensión } & Módulo de Young & $207000 \mathrm{MPa}$ \\
& Coeficiente de Poisson & 0,33 \\
& Módulo cortante & $77819,5 \mathrm{MPa}$
\end{tabular}

Las restricciones son necesarias para el estudio porque permiten colocar condiciones de fijación en algunos elementos que así se comporten, teniendo en cuenta, el tipo de análisis que se realiza. En el estudio se aplicaron restricciones de tipo fijas, la primera en la superficie inferior de las estructuras que soportan el prototipo. En la segunda se fijan la base del motoreductor, la base de una chumacera y el elemento separador entre ellas debido a que están sujetas entre sí.

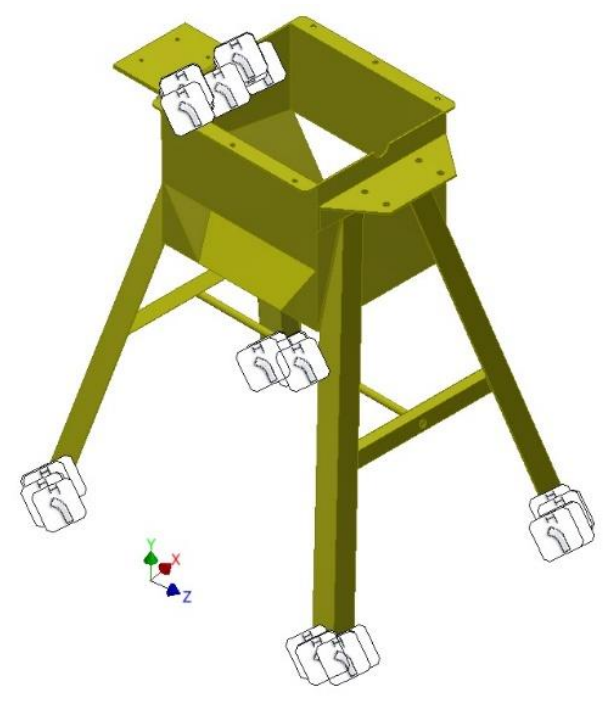

Figura 4. Restricciones del modelo. 
En la figura 4 se pueden visualizar donde se posicionan las restricciones del modelo, las mismas se simularon fijas en todos los ejes, de igual manera se utilizaron las mismas restricciones para la placa soporte del moto-reductor.

Las cargas que soporta el diseño permiten conocer el comportamiento del material y a su vez de las estructuras o elementos que lo componen. En el presente trabajo se aplicaron las cargas teniendo en cuenta, la fuerza que ejercen los elementos superiores. En la tabla 3 se pueden observar los valores obtenidos de las masas de los elementos.

Tabla 3. Valores de las cargas de los elementos superiores.

\begin{tabular}{cc}
\hline Tipo de carga & Magnitud \\
\hline Fuerza 1 & $98,96 \mathrm{~N}$ \\
Gravedad & $9,81 \mathrm{~N}$ \\
Fuerza 2 & $189,483 \mathrm{~N}$ \\
Fuerza 3 & $42,052 \mathrm{~N}$ \\
\hline
\end{tabular}

En la figura 5 se pueden observar las posiciones de las fuerzas simuladas en el modelo del prototipo de máquina separadora de partículas ferromagnéticas. La Fuerza 1 se puede ver en color azul, la misma simula el peso de la parte superior que incluye solamente la tapa y la tolva de alimentación. La Fuerza 2, color rojo, se aplica sobre los soportes laterales de las chumaceras. La Fuerza 3, color verde, se aplica en la base del moto-reductor teniendo en cuenta la masa del mismo. Por otra parte, está la gravedad que se aplica teniendo en cuenta la posición del modelo como fuerza natural.

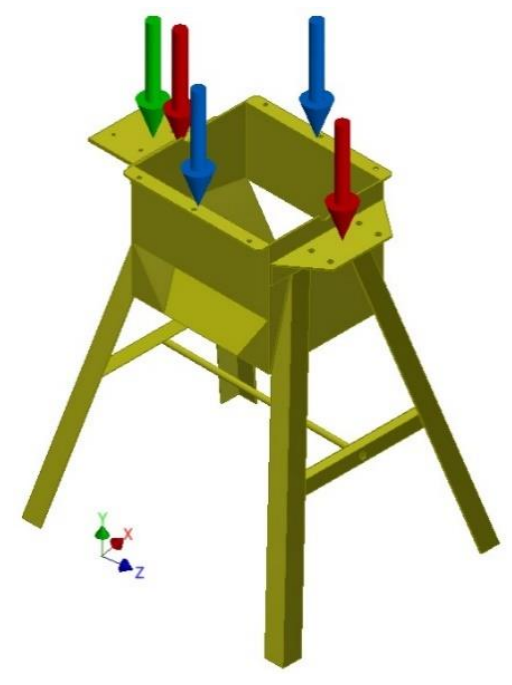

Figura 5. Posicionamiento de las cargas sobre el modelo.

El proceso de mallado se hace necesario para el cálculo el análisis por elementos finitos, de la calidad de la malla y del modelo depende el resultado del estudio. El software solamente propone un tipo de malla tetra 10 que tiene 4 puntos físicos y 10 nodos para la interpolación, además sus elementos son volumétricos. En la tabla 4 se muestran los datos de la malla utilizada en los cálculos. 
Tabla 4. Propiedades de la malla.

\begin{tabular}{cc}
\hline Configuraciones & Magnitud \\
\hline Tamaño medio de elemento (fracción del diámetro del modelo) & 0,1 \\
Tamaño mínimo de elemento (fracción del tamaño medio) & 0,2 \\
Factor de modificación & 1,5 \\
Ángulo máximo de giro & $60^{\circ}$ \\
Cantidad de nodos & 251229 \\
Cantidad de elementos & 124149 \\
\hline
\end{tabular}

Además del proceso de creación de la malla, existe la convergencia del mallado, dicho factor es importante pues permite obtener una solución precisa, adaptando la malla con diferentes densidades y comparando los resultados. El procedimiento se puede realizar manual pero el software tiene la opción de configuración de convergencia donde se aplica el método $\mathrm{h}$ adaptativo. Las condiciones aplicadas al estudio se pueden observar en la tabla 5.

Tabla 5. Características de la convergencia del mallado.

\begin{tabular}{cc}
\hline Configuraciones & Magnitud \\
\hline Número máximo de refinados $\mathrm{h}$ & 10 \\
Criterio de paradas & $5 \%$ \\
Umbral de refinado $\mathrm{h}$ & 0,750 \\
\hline
\end{tabular}

\section{RESULTADOS Y DISCUSIÓN}

En el presente epígrafe se muestran los resultados del diseño y análisis por elementos finitos del prototipo de máquina de separación de partículas ferromagnéticas. Luego de los resultados del cálculo de los parámetros funcionales de la máquina, se presenta como elección un motor de $0,25 \mathrm{HP}$ para el uso de la misma.

Asimismo, en la figura 6, se muestran los resultados del cálculo de las tensiones de Von Mises donde se obtuvieron valores máximos de 49,1 $\mathrm{N}$ muy inferiores al valor del límite elástico del material. Lo anterior refiere a que el material utilizado soporta las cargas simuladas.

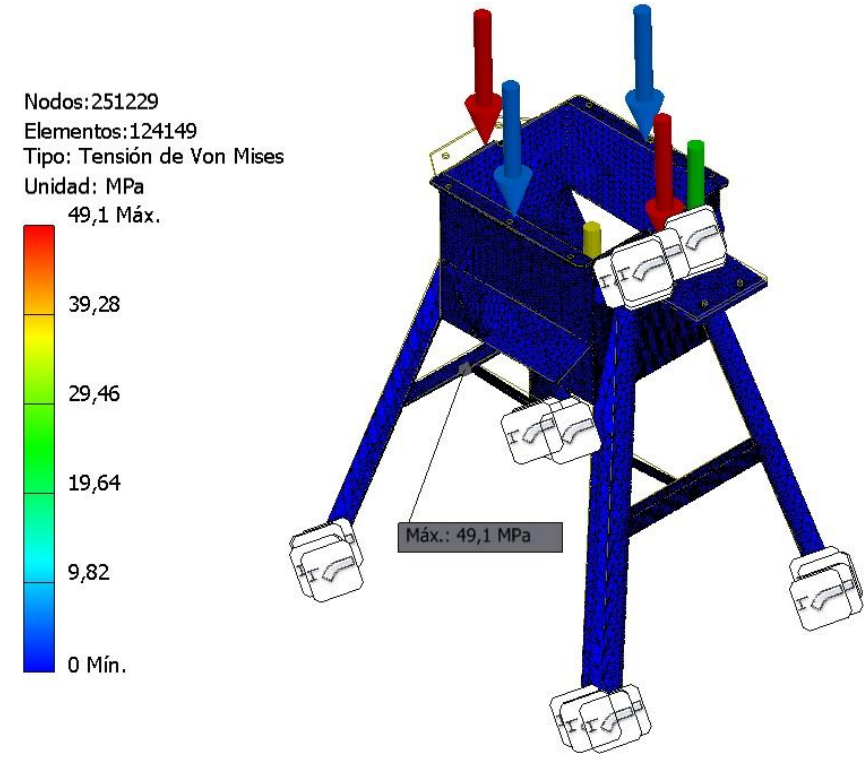

Figura 6. Resultados de las tensiones de Von Mises. 
Además, se obtuvieron los resultados para el desplazamiento resultante que como se observa en la figura 7, el máximo valor se encuentra en el soporte de las chumaceras con $7,7 \cdot 10^{-5} \mathrm{~m}$ de desplazamiento. Los resultados demuestran que no existen deformaciones debido a que son valores pequeños que no se detectan a simple vista.

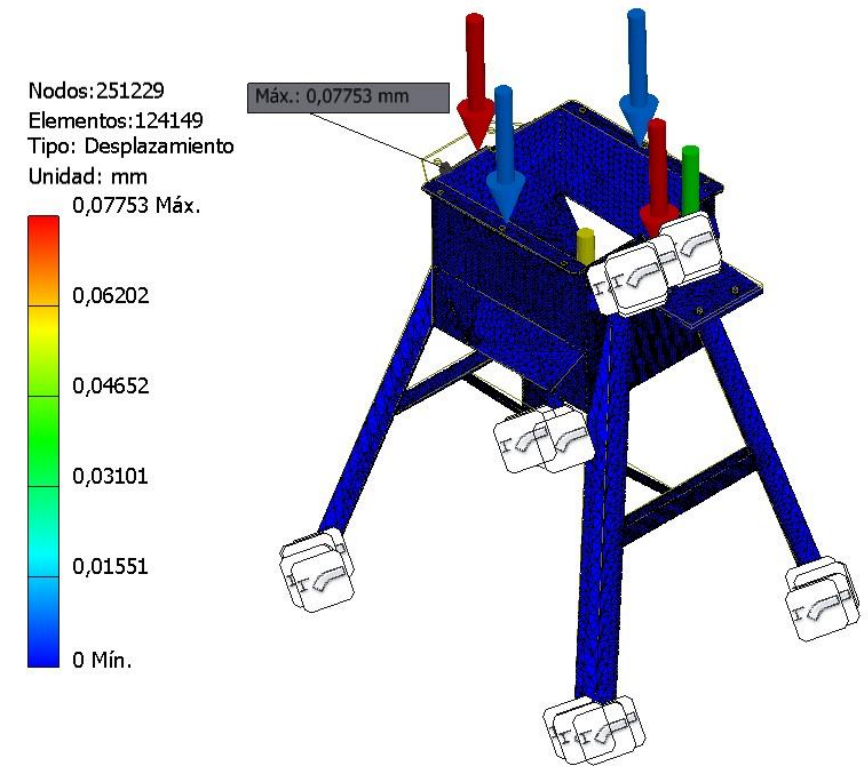

Figura 7. Desplazamientos resultantes.

Por último, se obtuvieron los resultados del coeficiente de seguridad. En la figura 8 se puede observar que los valores están entre 5,6 y 15, esto demuestra que la relación entre tensión última y tensión admisible están distantes. También se puede decir que el valor de esfuerzo máximo está muy distante de la resistencia a la fluencia del material.
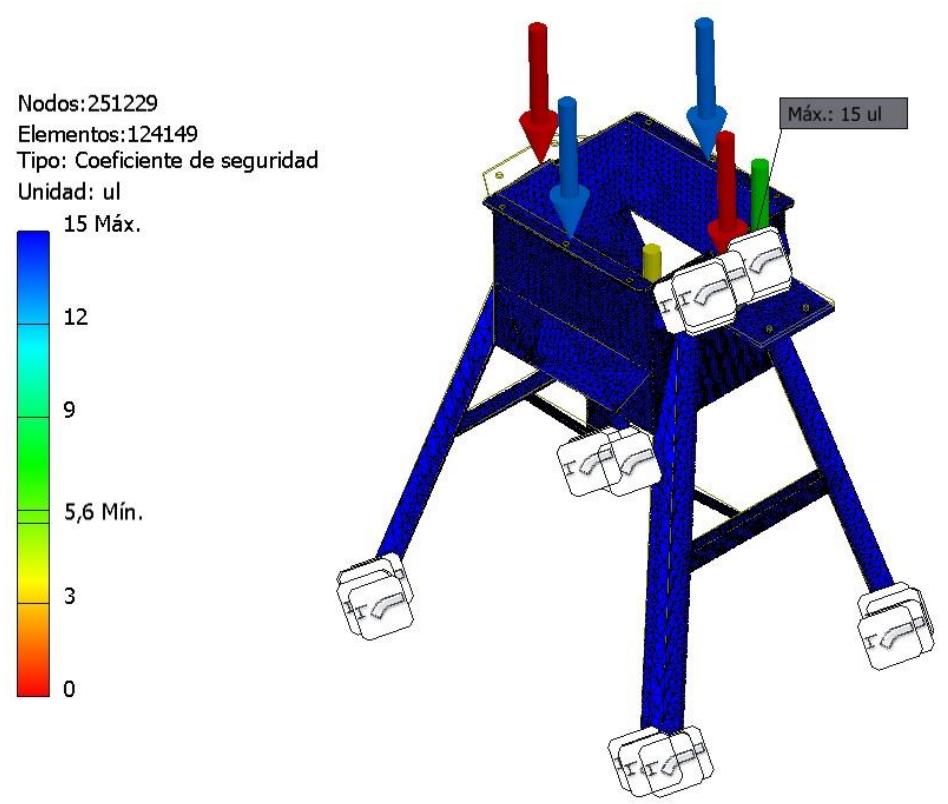

Figura 8. Coeficiente de seguridad. 
Los valores de coeficiente de seguridad permiten conocer si el modelo es seguro, para ello los resultados deben ser mayores o iguales que 1, quizás cercanos. Sin embargo, en el presente trabajo se puede deducir que el material utilizado está sobredimensionado por los altos valores. Esto no quiere decir que el estudio se encuentre incorrecto, sino que con la aplicación del acero AISI 1020 se podría estar realizando un gasto excesivo. Por lo tanto, se puede realizar el mismo estudio aplicando otro tipo de material que permita una comparación del comportamiento mecánico en ambos.

\section{CONCLUSIONES}

Con los resultados obtenidos se llegan a las conclusiones de la investigación, donde se seleccionó el método de separación mediante tambor magnético de imanes permanentes. El diseño del prototipo de máquina está basado en una planta de procesamiento de pequeña escala, por tales razones sus dimensiones se ajustan a la demanda del cantón La Maná. Por otra parte, se realizaron los cálculos de los parámetros funcionales de los elementos motrices lo que permitió seleccionar un motor de 0,25 HP. Además, los resultados del cálculo por elementos finitos permitieron conocer que tanto las tensiones de Von Mises y el desplazamiento resultante presentaron valores excelentes, sin embargo, el coeficiente de seguridad estuvo dentro de valores exagerados para este tipo de estructura por lo que se concluye que la misma está sobredimensionada y es recomendable realizar cambios en espesores y evaluar otros materiales.

\section{REFERENCIAS}

[1]. Dobrotă, D., Dobrotă, G., and Dobrescu, T., Improvement of waste tyre recycling technology based on a new tyre markings. Journal of Cleaner Production, 2020. 260 p.121141. doi: https://doi.org/10.1016/j.jclepro.2020.121141.

[2]. Topçu, İ. B. and Unverdi, A., 2 - Scrap tires/crumb rubber 2018, Woodhead Publishing ISBN: 978-0-08-102156-9.

[3]. Vallero, D. A., Chapter 8 - Effect of Waste on Ecosystems 2019, Academic Press ISBN: 978-0-12-815060-3.

[4]. Shulman, V. L., Chapter 26 - Tire Recycling 2019, Academic Press ISBN: 978-0-12815060-3.

[5]. Bi, Z., Finite Element Analysis Applications. A Sistematic and Practical Approach 2018,USA: Academic Press ISBN: 978-0-12-809952-0.

[6]. da Silva, P., Rodrigues, L., Lopes, S., Rebuzzi, M., and Proença, L., Chapter Three - Finite Element Modelling 2017, Butterworth-Heinemann ISBN: 978-0-12-813526-6.

[7]. Amé, R. M. and Lezama, D. H., Gestión de las discontinuidades geométricas combinadas en el diseño mecánico. Revista Cubana de Ingeniería, Concentración de tensiones; elementos finitos; diseño mecánico, 2012. 3(1): p.21-27.

[8]. Bárzaga, J., Morales, Y., Mena, E., Beltrán, R., and Morales-Leslie, J., Análisis numérico a un invernadero tipo túnel bajo cargas de vientos fuertes en Cuba. UTCiencia Ciencia y Tecnología al servicio del pueblo, cargas de viento; comportamiento estructural; elementos finitos; invernadero; tensiones, 2017. 4(2): p.62-70.

[9]. Emekli, N. Y., Kendirli, B., and Kurunc, A., Structural analysis and functional characteristics of greenhouses in the Mediterranean region of Turkey. African Journal of Biotechnology, 2010. 9(21): p.3131-3139. 
[10]. Estrada, R. and Gómez, E., Análisis del bastidor principal de la cosechadora de caña a través del Método de los Elementos Finitos. // Analysis of the main frame in cane combineharvester using the Finite Elements Method. Ingeniería Mecánica, 2006. 3(2006): p.61-68.

[11]. Li, S. and Feng, X., Study of structural optimization design on a certain vehicle body-inwhite based on static performance and modal analysis. Mechanical Systems and Signal Processing, 2020. 135 p.106405. doi: https://doi.org/10.1016/j.ymssp.2019.106405. 